\title{
Overturning instability in the mesosphere and lower thermosphere: analysis of instability conditions in lidar data
}

\author{
L. Hurd ${ }^{1}$, M. F. Larsen ${ }^{1}$, and A. Z. Liu ${ }^{2}$ \\ ${ }^{1}$ Department of Physics and Astronomy, Clemson University, Clemson, SC, USA \\ ${ }^{2}$ Department of Electrical and Computer Engineering, University of Illinois, Urbana, IL, USA
}

Received: 8 December 2008 - Revised: 7 April 2009 - Accepted: 14 July 2009 - Published: 24 July 2009

\begin{abstract}
Resonant sodium lidar measurements from the transition region between the mesosphere and lower thermosphere have revealed frequently-occurring overturning events characterized by vertical scales of $\sim 3-6 \mathrm{~km}$ and timescales of several hours. Larsen et al. (2004) proposed that a convective roll instability, similar to that found in the planetary boundary layer, is the likely mechanism responsible for the events. This type of instability requires an inflection point in the background winds near the center of the vortex roll with a low static stability region capped by an inversion. The earlier paper argued that the conditions required to support the instability are common in the altitude range where the features are found. In this paper, we use data from the University of Illinois sodium lidar that was located at the Starfire Optical Range near Albuquerque, New Mexico, and from the Maui/MALT Lidar Facility in Hawaii and present several cases that are used to examine the behavior of the inflection point in detail as a function of time during the evolution of the overturning event. In addition, we examine the background static stability conditions using the temperature data from the lidar.
\end{abstract}

Keywords. Meteorology and atmospheric dynamics (Thermospheric dynamics; Turbulence; Waves and tides)

\section{Introduction}

A number of observational studies of neutral instabilities in the mesosphere and lower thermosphere (MLT) region have focused on shorter time scale and smaller spatial scale features, most likely associated with gravity waves or KelvinHelmholtz instabilities in regions with low Richardson numbers. Several have shown evidence for both convective and

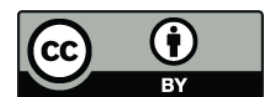

Correspondence to: L. Hurd (lhurd@clemson.edu) dynamical instabilities resulting from gravity wave fluctuations in the mesosphere (e.g., Fritts and Rastogi, 1985; Bishop et al., 2004). Larsen et al. (2004), however, suggested that there is also a different class of overturning features with longer time scales of several hours and larger vertical scales of $4-6 \mathrm{~km}$ that are not easily explained by gravity wave or Kelvin-Helmholtz instabilities. In that paper Larsen et al. argued that the relevant dynamics were closely related to the inflection point instabilities that generate convective roll vortex features in the planetary boundary layer (PBL) with characteristics similar to those observed in the MLT region.

The literature on convective roll features in the PBL is extensive (see, e.g., Brown, 1972, 1980; Stensrud and Shirer, 1988 , and references therein). Originally, it was thought that the rolls were infrequent since the events were mainly identified by the visual indicator of cloud streets observed from aircraft or in satellite photos. More recent observations using radars and lidars have shown that the features are common and dynamically important in the boundary layer. The features, which are usually referred to as boundary layer rolls or vortex rolls, are associated with an instability that requires an inflection point in the background wind profiles that leads to longitudinal instabilities. The inflection point is the height where the second derivative with height of the wind component vanishes, i.e., the altitude where the Rayleigh inflection point criterion for instability is satisfied. In addition to the sheared wind profile, the existence of a lower stability region capped by a region of higher stability is also required to generate the rolls. As discussed in more detail by Larsen et al. (2004), the instability is distinct from the Kelvin-Helmholtz instability and does not require subcritical Richardson numbers.

Lilly (1966) presented the first detailed analysis of the inflection point instability as it applies to the Ekman spiral in the PBL. In particular, he showed that the variation of the winds with height in the lowest few kilometers of the atmosphere tends to be unstable because of the turning and

Published by Copernicus Publications on behalf of the European Geosciences Union. 

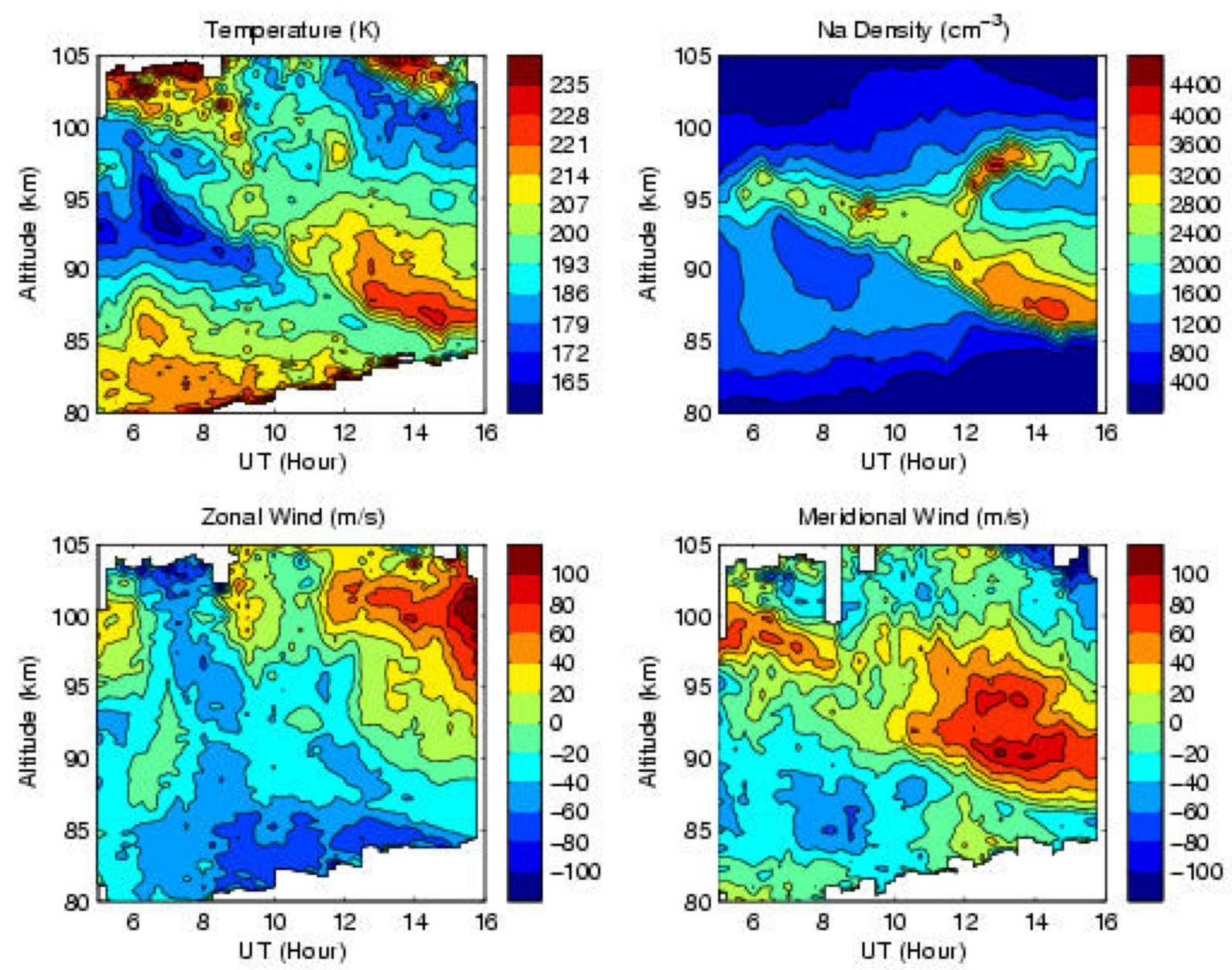

$\mathrm{Na}$ Wind/Temperature Lidar, Maui, $\mathrm{HI}$

April 13, 2002

Fig. 1. Contour plots as a function of time and altitude of the temperature, sodium density, zonal winds, and meridional winds measured with the Maui/MALT lidar on 13 April 2002.

wind speed shears that generally characterize that altitude range. The background winds therefore often satisfy the requirement of the existence of an inflection point together with the requirement for a substantial shear across that inflection point. Analytic theories, such as that developed by Lilly, have been successful in showing the tendency for instability, but have been less successful in predicting the characteristics of the structures. Numerical models are generally required because of the large number of variables involved. Moreover, the instability depends on the angle between the mean flow and the roll orientation and on the horizontal wavelength. Results of numerical modeling studies have predicted aspect ratios, i.e., the ratio of the horizontal to the vertical scale of the rolls, of 5-6, although aspect ratios as large as 15 have been observed in the boundary layer. Both the analytic and numerical studies predict that the convective roll axes will be rotated clockwise between $\sim 16-20^{\circ}$ in the horizontal plane relative to the mean background wind direction at the height of the inflection point. The convective rolls are important in the PBL because of the enhanced transport and eddy mixing that they create.

The overturning events of interest to us occur at the mesosphere/lower thermosphere transition region near $100 \mathrm{~km}$ altitude rather than in the boundary layer. In the upper atmosphere, the inflection point instability is associated with the turning and speed shears associated with the tidal fluctuations and the rapid increase in the wind speeds that occurs in the altitude range between 95 and $105 \mathrm{~km}$, as discussed by Larsen (2002), rather than the Ekman spiral. In addition, the transition region is characterized by lower static stability in the mesosphere capped by a higher static stability region at the mesopause and in the lower thermosphere, i.e., the second condition required to support the convective roll instability. 

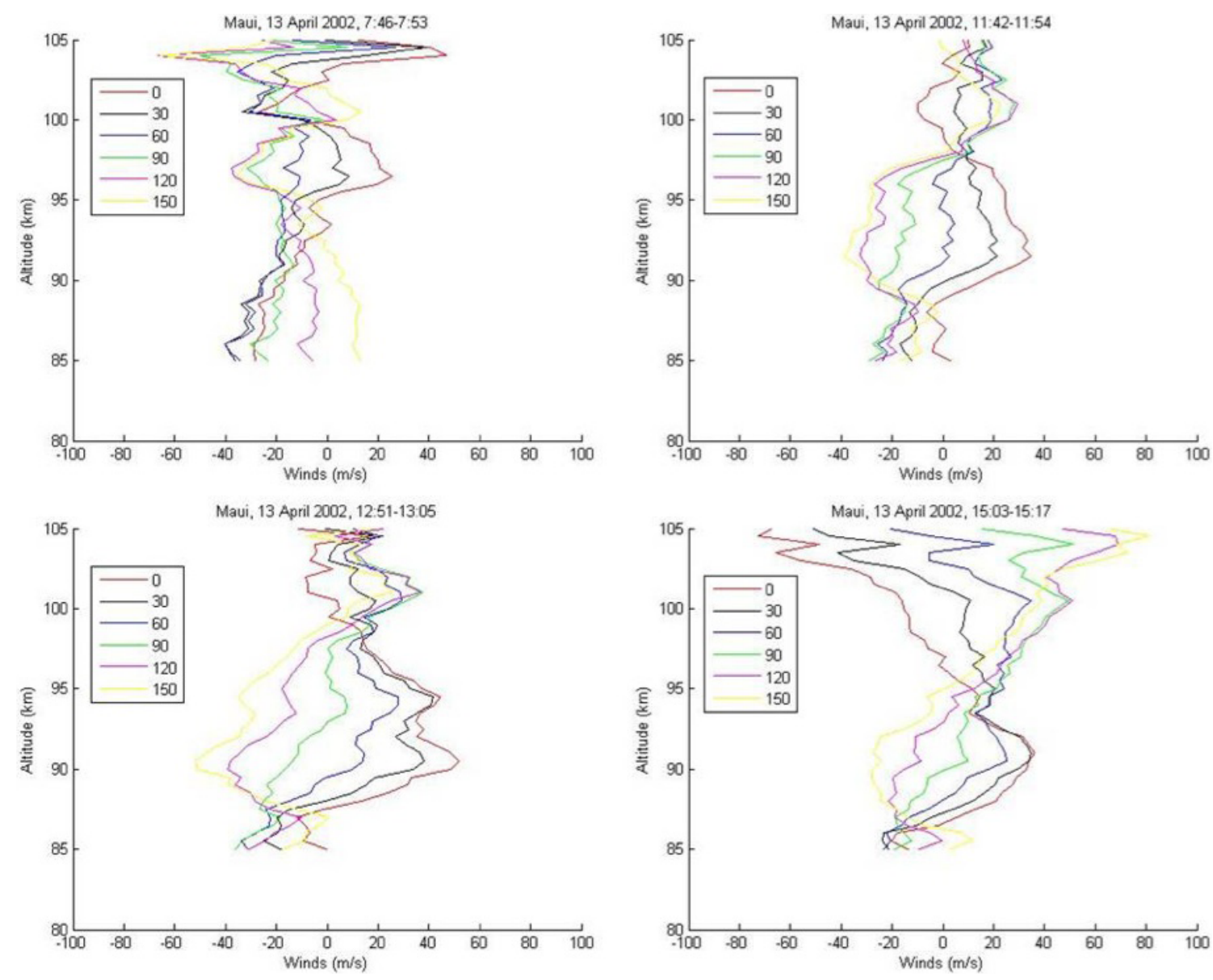

Fig. 2. Wind profiles showing shears and and the inflection point for varying vertical orientations at different stages of the observing period for 13 April 2002. The legend refers to the degrees of the direction for the corresponding profile.

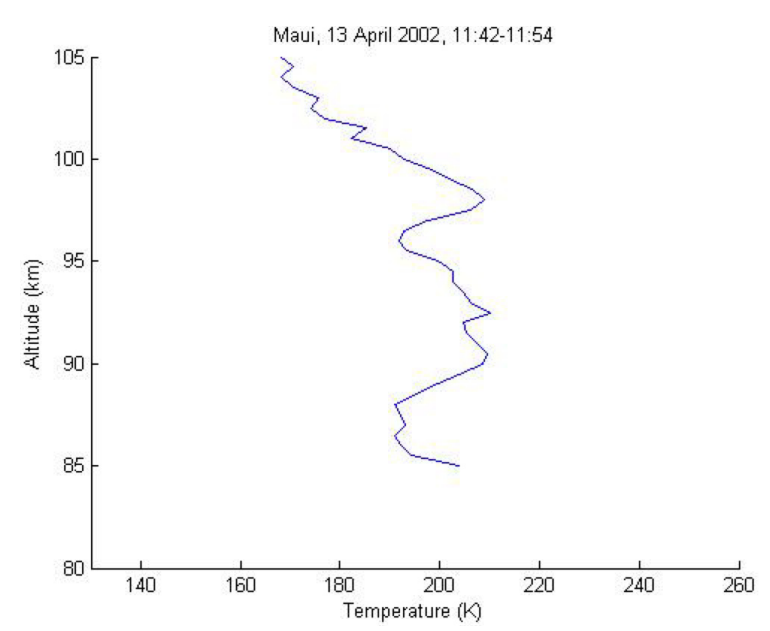

Fig. 3. Temperature profile averaged for about $15 \mathrm{~min}$ during the overturning event on 13 April 2002.
In this paper we examine data from several cases obtained with the high-sensitivity University of Illinois sodium lidars that were operated at the Starfire Optical Range in New Mexico and at the Maui/MALT facility at Haleakala on the island of Maui in Hawaii. Besides the sodium density, the instruments provide the temporal variations of the temperature and wind profiles. Details of the lidar instrumentation and the technique can be found in the article by Liu et al. (2002). The overturning features are characterized by a vertical scale of $\sim 4-6 \mathrm{~km}$ near the transition from the mesosphere to the lower thermosphere. The observations also show a timescale of $\sim 1-4 \mathrm{~h}$. Our goal here is to determine if the observed characteristics are consistent with the behavior expected for a convective roll instability. Specifically, we will estimate the aspect ratios of the convective rolls. This will allow us to make comparisons with the convective roll studies from the lower atmosphere, described above, and to look for similarities between the vortex rolls that are commonly observed in the planetary boundary layer and the convective rolls that are observed in the lidar data. As we will show, the aspect ratio 

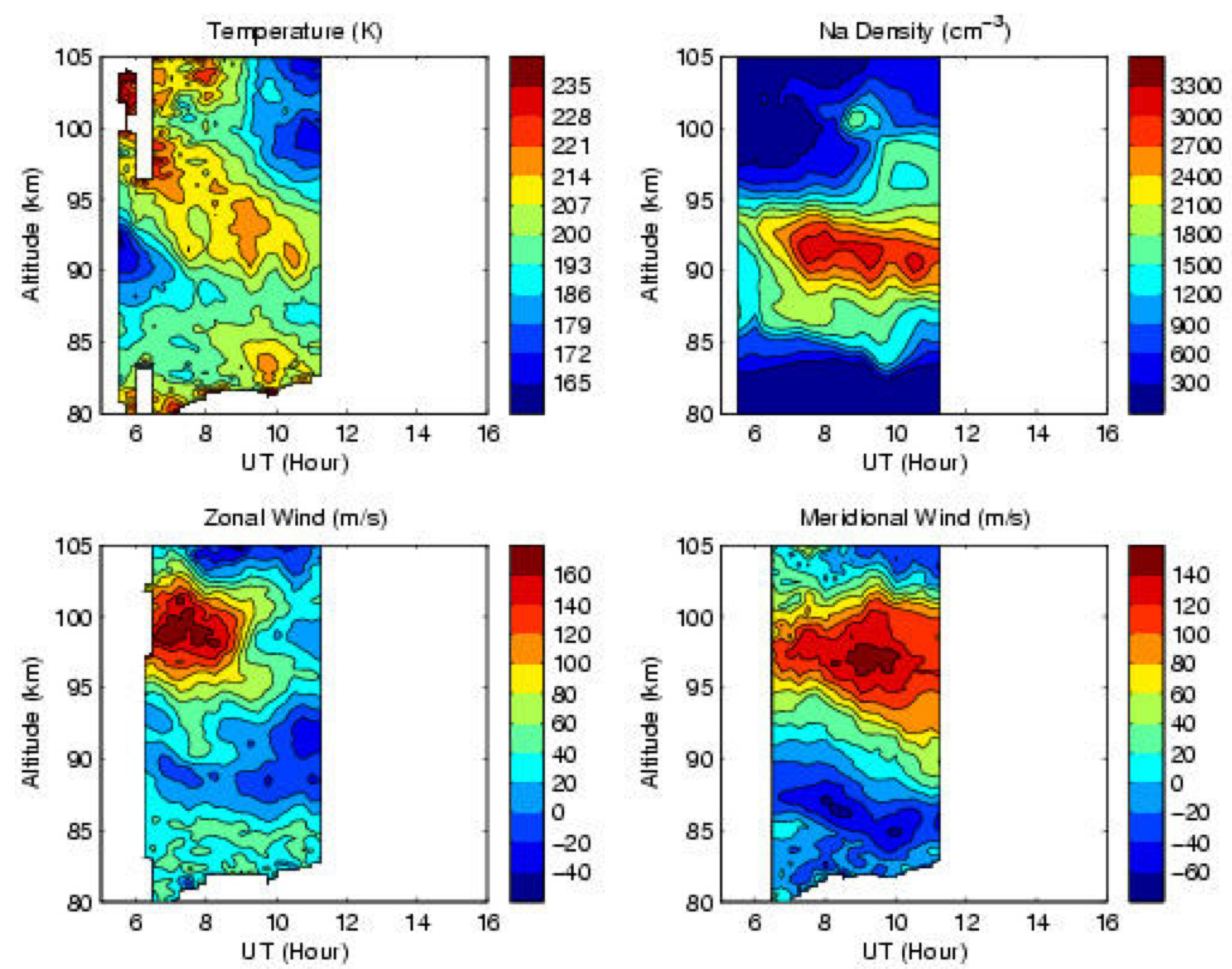

Na Wind/Temperature Lidar, Maui, HI

January 14, 2002

Fig. 4. Contour plots as a function of time and altitude of the temperature, sodium density, zonal winds, and meridional winds measured with the Maui/MALT lidar on 14 January 2002.

for the MLT events is consistent with the aspect ratio predictions of the modeling studies for the lower atmosphere. This analysis also allows us to investigate the variation of the wind profile inflection point with time as the overturning features develop and eventually dissipate. As we will show, the roll features are present during the period when the inflection point is present and well defined, but not before or after.

The analysis presented here does not exclude other instabilities as an explanation for the observed roll structures, but shows that the convective roll instability can explain the main characteristics of the observed rolls. Particularly, we will present an analysis of three events that are representative of other similar events.

\section{Observations}

\subsection{Maui/MALT, 13 April 2002}

The first example of a convective roll observed with the Maui/MALT lidar is shown in Fig. 1 from 13 April 2002. The sodium density plotted in the upper righthand panel of the figure shows that overturning began near 12:00 UT around $94-95 \mathrm{~km}$. The top of the structure was at $98-99 \mathrm{~km}$ and extended to the end of the observing period near 16:00 UT. The temperatures, zonal winds, and meridional winds measured by the lidar are plotted in the other three panels in the figure.

A necessary condition for the convective roll instability is that the second derivative of the background wind with respect to height is zero somewhere, i.e., that there is an inflection point in the background wind profile (see, e.g., Brown, 1972, 1980; Etling and Brown, 1993). In practice, the roll axes are expected to be aligned within $16^{\circ}-20^{\circ}$ of the direction along which the largest shear occurs with an inflection point. The text by Houze (1994) presents a good discussion 

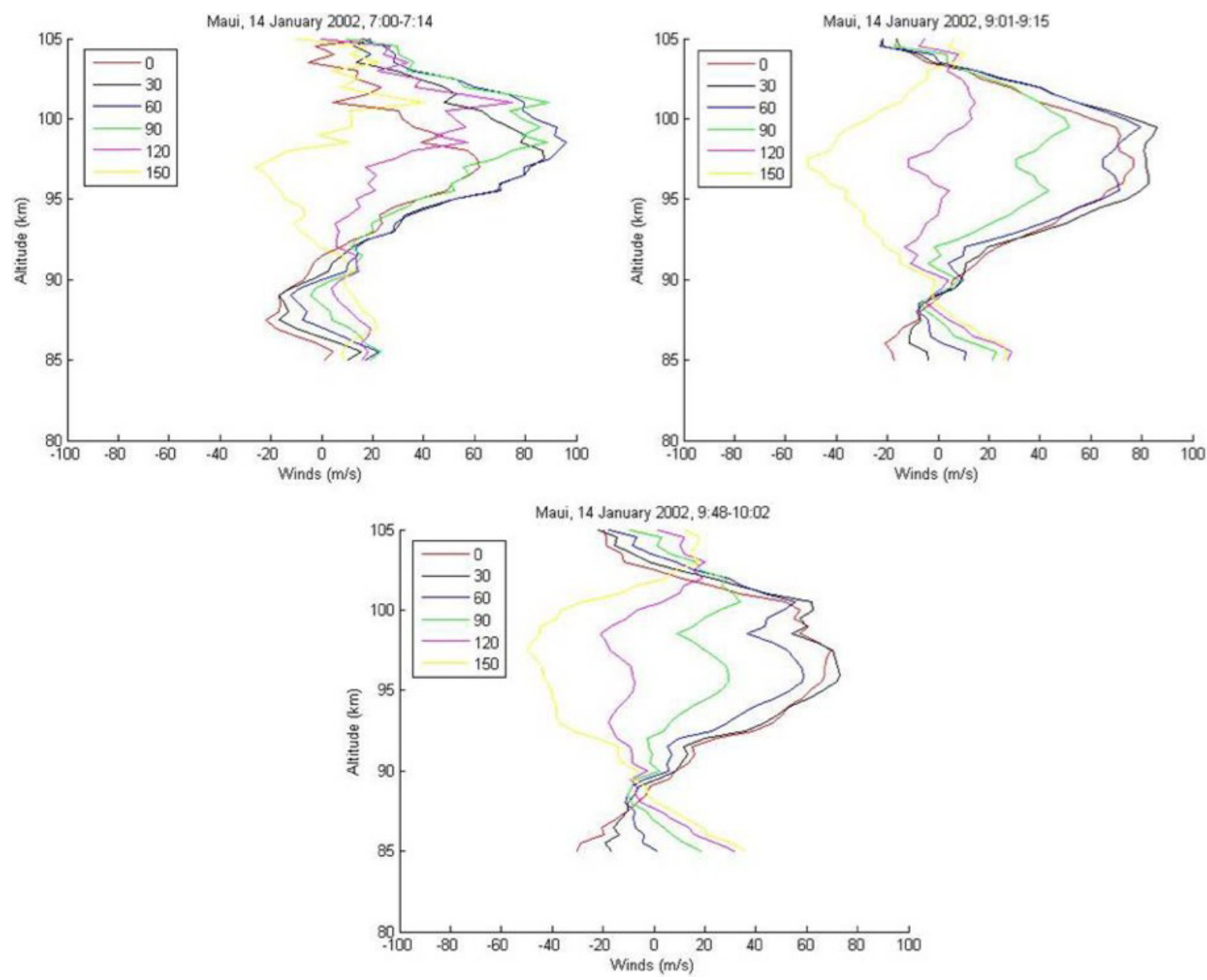

Fig. 5. Wind profiles showing shears and and the inflection point for varying vertical orientations at different stages of the observing period for 14 Janurary 2002. The legend refers to the degrees of the direction for the corresponding profile.

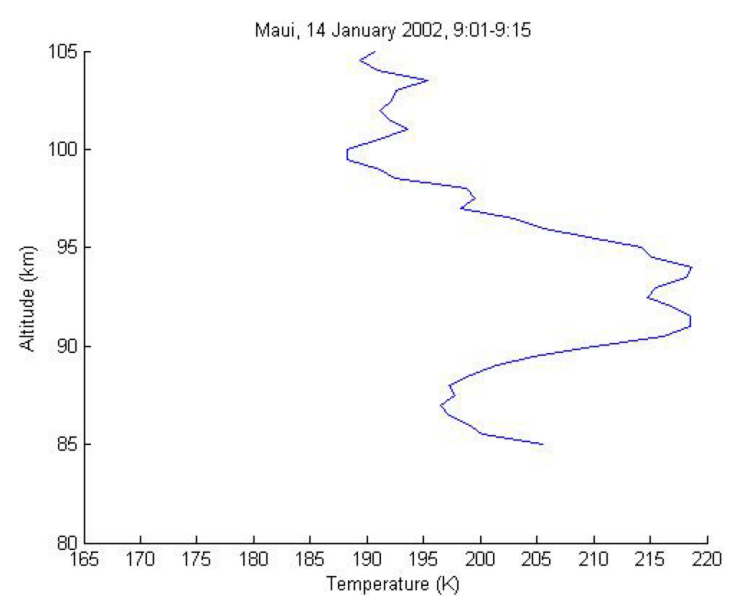

Fig. 6. Temperature profile averaged for about 15 min during the overturning event on 14 January 2002. with an example of how the wind profiles are analyzed in the case of a boundary layer flow with an Ekman spiral profile. In effect, for variations that are close to sinusoidal, the inflection point is found near the zero crossing.

In Fig. 2, we have plotted average wind profiles for four different times during the overturning event, using the same approach described by Houze. Specifically, the wind profile components for planes separated by $30^{\circ}$ in azimuth are shown for the azimuth range from $0^{\circ}$ to $150^{\circ}$. The four sets of profiles correspond to times near 07:50, 11:50, 13:00, and 16:10 UT, i.e., a period before the overturning starts, a period at the start of the overturning, a period when the overturning is fully developed, and a period at the end of the observation interval. The profiles from 11:50 UT show a clearly developed zero crossing in the center of the altitude range where the roll structure develops. The zero crossing is still present, although not as well defined in the profile from 13:00 UT, and disappears completely from the height range of the convective roll by the end of the observation period when the overturning feature becomes weaker and appears to dissipate. 
From the figure, it is evident that the largest shear occurs along the $150^{\circ}$ azimuth direction. The rolls are expected to be long, extended structures in the direction along that plane. If the roll axis was exactly aligned with the mean wind direction, no time variation would be expected in the structure observed from a ground-fixed position, but since the roll axis alignment is slightly to the right of the geostrophic wind direction, the small wind component spanwise to the roll slowly advects the roll structure past the observing site. In our case, this angle $\phi$ is assumed to be $18^{\circ}$. The overturning takes place over a period of $\sim 4 \mathrm{~h}$. The magnitude of the wind velocity along the $150^{\circ}$ plane is $\sim 4 \mathrm{~m} / \mathrm{s}$. Using these values gives a horizontal scale size for the vortex roll around $18 \mathrm{~km}$. Combined with the vertical scale size of $4 \mathrm{~km}$, we get an aspect ratio of about 4.5 , which is in qualitative agreement with the aspect ratios for rolls in the planetary boundary layer.

The temperature profile close to the time when the roll feature first develops is shown in Fig. 3. A temperature inversion is evident above the altitude where the roll develops. The altitude range below the inversion is characterized by a negative lapse rate and lower stability. The event, therefore, has the lower stability region capped by a higher stability region that is a characteristic of the convective roll instability.

\subsection{Maui/MALT, 14 January 2002}

The second overturning event analyzed here is from 14 January 2002. The height/time contours of the sodium density are shown in the upper righthand section of Fig. 4. The layer peak value is about $3300 \mathrm{~cm}^{-3}$ and occurs around 08:00 UT at an altitude near $92 \mathrm{~km}$. The feature of interest, however, is the overturning event that occurs between 07:00 and 10:00 UT. The bottom of the overturning is near $95 \mathrm{~km}$ and is observed soon after 10:00 UT. The top of the convective roll is located near $103 \mathrm{~km}$ and was observed close to 07:00 UT. Therefore, the feature in this case has a duration of $3-4 \mathrm{~h}$ and a vertical scale of $5-6 \mathrm{~km}$. The temperatures, zonal winds, and meridional winds measured by the lidar are also shown in the other three panels of Fig. 4 in a format similar to that in Fig. 1.

In this case, the sense rotation associated with the convective roll is reversed, so that the top extent of the feature is observed first. The instability is expected to produce pairs of counter-rotating vortices. The rotation therefore depends on which vortex tube in the pair happens to be above the location of the lidar at the time of the observation.

As in the previous example we can use the wind profiles to determine the orientation of the convective vortex axes. The winds for the 14 January event are plotted in Fig. 5 in the same format that was used in the analysis in the previous section. We also show in this case the winds at a time when the overturning is fully developed (upper righthand panel), as well as at the beginning and end of the observed overturning event. In general, we are assuming that the vortex roll feature is traced out as the roll structure is advected past the lidar by the spanwise wind component, which is usually small. In other words, we assume that the structure is frozen in the flow to some extent. In the example in the previous section, it was clear however that the temporal variations are also important in determining when the appropriate conditions occur to initiate the instability and when the conditions that support the instability disappear. The competing roles of temporal and spatial variations is even more apparent in the 14 January example. In this case, the inflection point is evident in the later wind profiles (lower panel in Fig. 5) with a strong shear across the inflection point. An inflection point is also evident in the wind profile from the time when the overturning is well developed (upper righthand panel). The inflection point is not well-defined in the earliest profile (upper lefthand panel) that corresponds to a time when the overturning feature had weakened.

The largest shear in the winds occurred along the $60^{\circ}$ orientation in this case. Therefore, the convective roll should align along a direction approximately $18^{\circ}$ to the right of that direction. To determine the horizontal scale of the overturning, we again use the time scale of the overturning and the magnitude of the spanwise velocity at the inflection point to estimate the horizontal scale of the convective roll and the aspect ratio. In this case the overturning occurred over a period of $3 \mathrm{~h}$, and the winds plotted in the righthand plot of Fig. 5, which represent the conditions during the event, give a spanwise velocity of $\sim 5 \mathrm{~m} / \mathrm{s}$. The horizontal scale for the roll is therefore $\sim 17 \mathrm{~km}$. Since the vertical scale is close to $6 \mathrm{~km}$, the aspect ratio is about 3 . These values are qualitatively consistent with those found in the planetary boundary layer.

The average temperature profile for the 09:00 to 09:15 UT interval is shown in Fig. 6. Again, the vertical temperature structure shows that the requirement for a lower static stability region capped by a higher static stability region is fulfilled.

\subsection{Starfire, 26 October 2000}

The final example is from observations with lidar at the Starfire Optical Range operated during the Turbulent Oxygen Mixing Experiment (TOMEX) on 26 October 2000. The contour plots of the winds and temperature are shown in Fig. 7. The overturning feature occurred between 06:00 and 10:00 UT. The bottom of the structure was located near $95 \mathrm{~km}$ at 08:00 UT, and the top was near $101 \mathrm{~km}$. The strong upwelling associated with the overturning is evident between 09:00 and 10:00 UT. The feature had a timescale of 3-4 h and a vertical scale of $5-6 \mathrm{~km}$. The sense rotation of the convective roll is the same as the previous example.

Once again, the wind profiles are used to determine the roll axis orientation and are presented in Fig. 8. As in the previous cases, the development of the inflection point was clear as the overturning developed. The largest shear in the winds occurred along the $150^{\circ}$ orientation, implying that the 

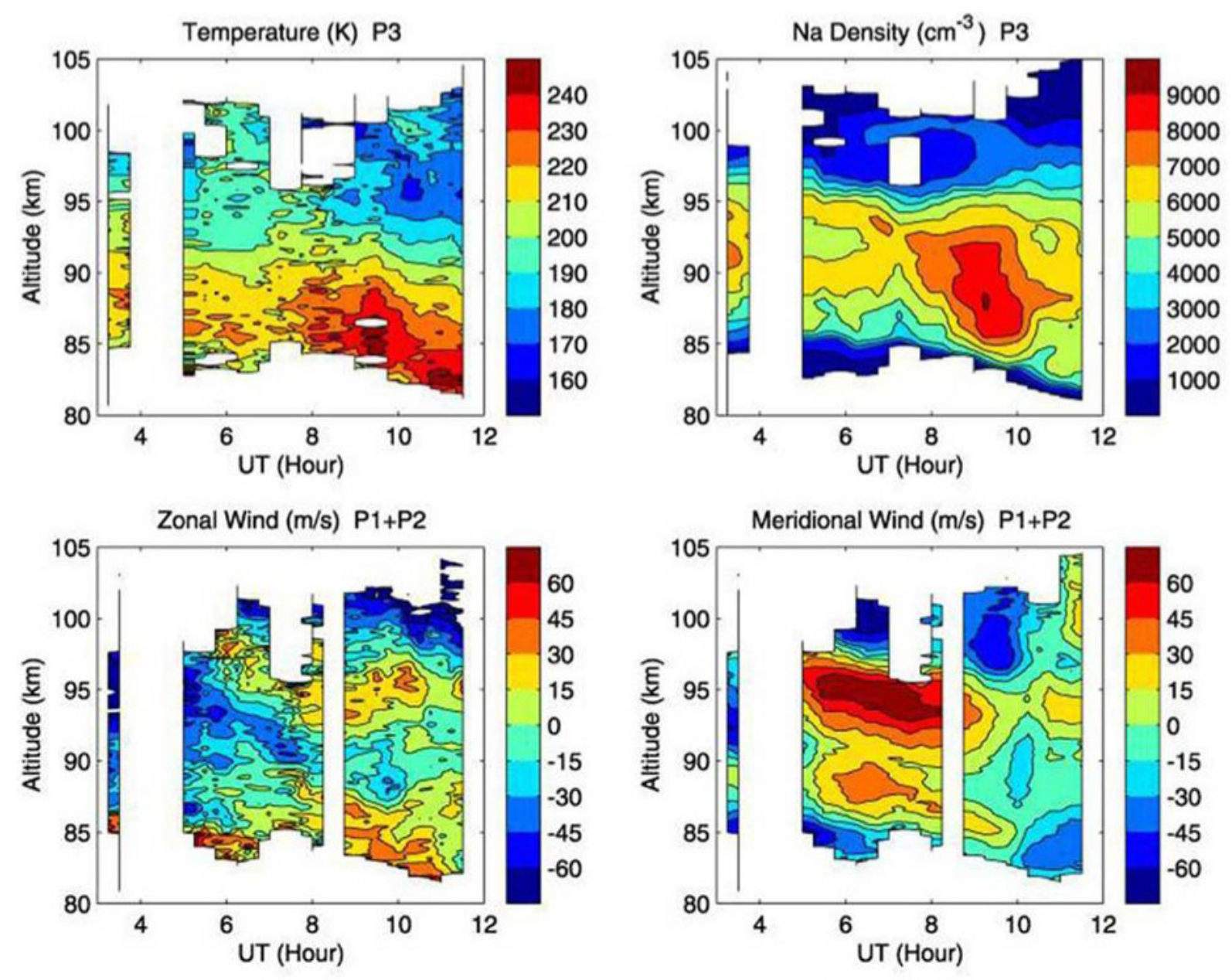

Na Wind/Temperature Lidar, SOR, NM

Oct 26, 2000

Fig. 7. Contour plots as a function of time and altitude of the temperature, sodium density, zonal winds, and meridional winds measured with the Starfire lidar on 26 October 2000.

convective roll was aligned approximately $18^{\circ}$ to the right of that direction. The spanwise velocity calculated from the wind profiles is $\sim 4 \mathrm{~m} / \mathrm{s}$. This result, and the period of $\sim 3.5 \mathrm{~h}$, yields a horizontal scale of $\sim 16 \mathrm{~km}$. As discussed above, the vertical scale is near $5 \mathrm{~km}$, which results in an aspect ratio of about 3 for this convective roll. Similar to the previous examples, the values are qualitatively consistent with those found in the planetary boundary layer.

The average temperature profile for the time near 10:00 UT is presented in Fig. 9. The transition from lower static stability to a higher static stability is again evident.

\section{Conclusions}

The three examples presented here show an overturning structure in the sodium density that has characteristics consistent with the expectations for an inflection-point insta- bility mechanism, similar to the convective roll instabilities observed in the planetary boundary layer. The overturning structures appear in the region where the inflection point in the wind profiles becomes well defined and weaken or disappear when either the inflection point disappears or the shear across the inflection point weakens. The aspect ratios, i.e., the ratio of the spanwise horizontal scale to the vertical scale, are also consistent with the aspect ratios for such features observed in the lower atmosphere. In all three cases, the requirement for a higher stability region overlying a lower stability region, which is conducive to the development of a convective roll, was also found to exist.

The convective roll structures have a relatively large spanwise horizontal scale that is estimated to be on the order of $15-20 \mathrm{~km}$ in the cases presented here. Since the roll axes are aligned close to the mean wind direction, the spanwise wind component is small, leading to the long time scale of 

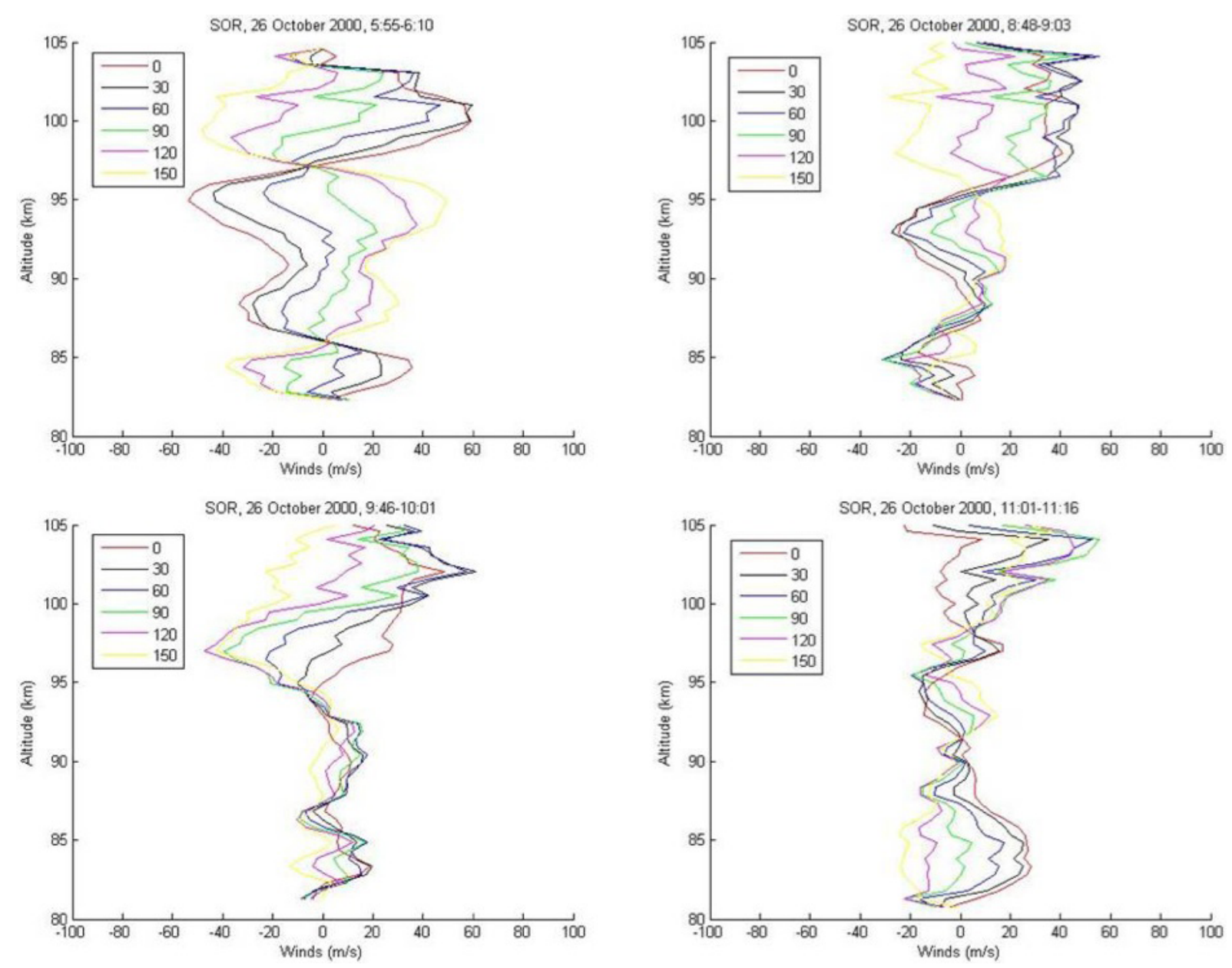

Fig. 8. Wind profiles showing shears and and the inflection point for varying vertical orientations at different stages of the observing period for 26 October 2000. The legend refers to the degrees of the direction for the corresponding profile.

the features. With a single-station observation, such as those presented here, it is impossible to separate the temporal and spatial variations unambiguously. In the first case from 13 April 2002, the sense of rotation of the overturning feature and time variation of the flow suggest an interpretation in which the conditions for instability develop with time and then disappear later, along with the overturning. The other two cases, however, show weak instability conditions initially and stronger instability conditions later. In those cases, the strong upward movement occurs later, i.e., at the time when the stronger instability conditions are observed, suggesting that the instability conditions characterize the region rather than the specific time. The development of the instabilities in any given case is likely to be a combination of both effects, i.e., instability conditions that develop both as a function of temporal variations in the background flow and as a function of the larger scale spatial gradients.

The analysis presented here is only sufficient to suggest that the convective roll instability can explain the characteris- tics of the observed features. The scale sizes of the rolls have the same aspect ratio as those of similar features in the lower atmosphere, and the rolls occur when the inflection point and stability conditions for roll features occur. A more convincing stability analysis requires a numerical model simulation of the MLT region with the appropriate wind and temperature profile conditions, although that is beyond the scope of the current study.

Overturning features of the type analyzed here, i.e., those with time scales of several hours and vertical scales of several kilometers are common in the lidar data sets from the Starfire Optical Range in New Mexico, from Puerto Rico, and from Maui/MALT in Hawaii, as discussed by Larsen et al. (2004) and Yount et al. (2009). The general characteristics shown by the analysis of the three events presented here appear to be representative of other events as well.

The convective roll structures in the planetary boundary layer are a common feature of the dynamics in that altitude range, and such large eddies contribute significantly to 


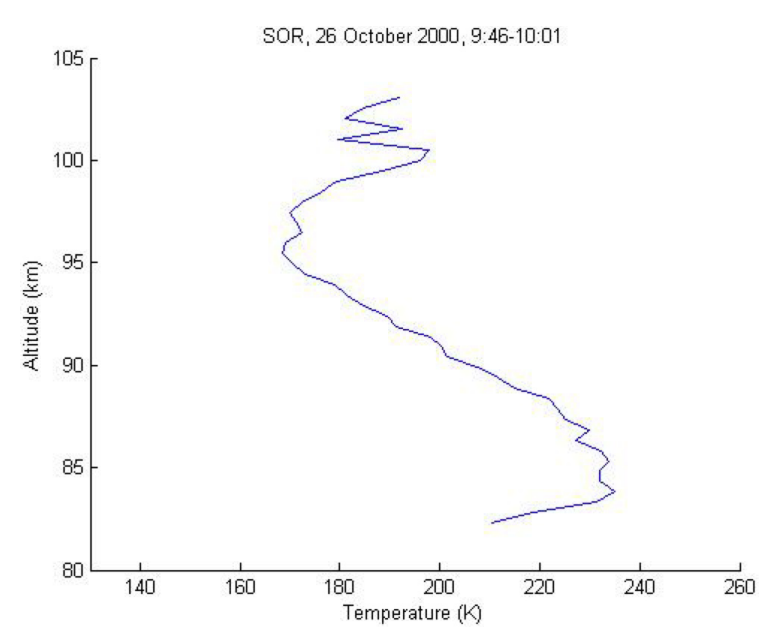

Fig. 9. Temperature profile averaged for about $15 \mathrm{~min}$ during the overturning event on 26 October 2000.

the mixing in the lower altitude range (see, e.g., Etling and Brown, 1993). The overturning features that we are attributing to a similar convective roll instability in the MLT region occur frequently in the lidar observations but detailed information about the spatial and temporal distribution of such features is still not known. Optical imager data could be helpful in determining the horizontal structure associated with the rolls since the vertical displacements are expected to produce signatures in the optical emissions if they occur in the emission layers (see, e.g., Xu et al., 2006). The most favorable conditions for the instability generally occur at heights above the green line emission layer, but it is likely that cases can be found in future studies in which both lidar and optical imager observations of the same roll structures are present. The instability conditions that were associated with the examples presented here are relatively common in that part of the atmosphere, which suggests that the overturning features may also be common, i.e., that they occur frequently and have a broad spatial distribution. If so, we would expect that large eddies of this type can contribute significantly to the mixing in this critical part of the atmosphere, as they do in the planetary boundary layer.

Acknowledgements. The work at Clemson was partially supported by NSF grant ATM-0541593 and NASA grant NNX07AJ99G. The lidar operation was supported by NSF grant ATM-0338425. The work at the University of Illinois was also supported by the Consortium of Resonance and Rayleigh Lidars under NSF grant ATM-0545704.
Topical Editor K. Kauristie thanks two anonymous referees for their help in evaluating this paper.

\section{References}

Bishop, R. L., Larsen, M. F., Hecht, J. H., Liu, A. Z., and Gardner, C. S.: TOMEX: Mesospheric and lower thermospheric diffusivities and instability layers, J. Geophys. Res., 109, D02S03, doi:10.1029/2002JD003079, 2004.

Brown, R. A.: On the inflection point instability of a stratified Ekman boundary layer, J. Atmos. Sci., 29, 850-859, 1972.

Brown, R. A.: Longitudinal instabilities and secondary flows in the planetary boundary layer: a review, Rev. Geophys. Space Phys., 18, 683-697, 1980.

Etling, D. and Brown, R. A.: Roll vortices in the planetary boundary layer: a review, Bound.-Lay. Meteor., 65, 215-248, 1993.

Fritts, D. C. and Rastogi, P. K.: Convective and dynamical instabilities due to gravity wave motions in the lower and middle atmosphere: Theory and observations, Radio Sci., 20, 1247-1277, 1985.

Houze Jr., R. A.: Cloud dynamics. Academic Press, pp. 164-173, ISBN-10: 0123568803, 1994.

Larsen, M. F.: Winds and shears in the mesosphere and lower thermosphere: Results from four decades of chemical release wind measurements, Geophys. Res. Lett., 107, 1215, doi:10.1029/2001JA000218, 2002.

Larsen, M. F., Liu, A. Z., Gardner, C. S., Kelley, M. C., Collins, S., Friedman, J., and Hecht, J. H.: Observations of overturning in the upper mesosphere and lower thermosphere, J. Geophys. Res., 109, D02S04, doi:10.1029/2002JD003067, 2004.

Lilly, D. K.: On the instability of Ekman boundary flow, J. Atmos. Sci., 23, 481-494, 1966.

Liu, A. Z., Hocking, W. K., Franke, S. J., and Thayaparan, T.: Comparison of $\mathrm{Na}$ lidar and meteor wind measurements at Starfire Optical Range, NM, USA, J. Atmos. Sol. Terr. Phys., 64, 31-40, 2002.

Stensrud, D. J. and Shirer, H. N.: Development of boundary layer rolls from dynamical instabilities, J. Atmos. Sci., 45, 1007-1019, 1988.

Xu, J., Smith, A. K., Collins, R. L., and She, C.-Y.: Signature of an overturning gravity wave in the mesospheric sodium layer: Comparison of a nonlinear photochemical-dynamical model and lidar observations, J. Geophys. Res., 111, D17301, doi:10.1029/2005JD006749, 2006.

Yount, C., Larsen, M. F., Friedman, J., Collins, R., and Liu, A. Z.: A study of occurrence frequency of convective rolls in the MLT region over four North American observation sites, Geophys. Res. Lett., submitted, 2009. 